\title{
Introduction: The FIA Research Roadmap, Priorities for Future Internet Research
}

\author{
Nick Wainwright and Nick Papanikolaou \\ Cloud and Security Lab, HP Labs \\ \{nick.wainwright, nick. papanikolaou\}@hp.com
}

\begin{abstract}
We describe the key findings of the Future Internet Assembly Research Roadmap for Framework Programme 8, which captures the ideas and contributions of the FIA community on the important research topics that should be addressed in future funding programmes. The findings of the roadmap have been produced through an open consultation of research projects who participate in FIA. It is primarily concerned with identifying research that can be carried out in the second half of this decade and which will have an impact in 2020 and beyond. By 'impact' we mean will result in products, services, systems, capabilities, that come to market and are available and deployed in that timeframe.
\end{abstract}

Introduction. We present here a vision for the Future Internet and its impact on individuals, businesses and society as a whole; the vision presented is based on an extended consultation carried out by the authors within the European Future Internet research community, as part of the work of the Future Internet Assembly (FIA).

The purpose of the consultation was to identify key challenges and research priorities for the Future Internet, particularly from the standpoint of current European research projects (in Framework Programme 7). The output of the consultation is documented in the form of a visionary research roadmap1.

In order to elicit inputs from members of the European Future Internet research community, we have had to actively participate in this community ourselves; the vehicle for doing so has been the EU Framework Programme 7 research project EFFECTSPLUS2, which carries out workshops and clustering activities for European projects, particularly in the area of ICT trust and security. As part of this Support Action we participate in and run aspects of the Future Internet Assembly (FIA).

The vision we present is intended to inform future research funding programmes, including the European Commission's "Horizon 2020" framework programme. We have validated the results of our initial consultation, and the associated vision, with a significant number of researchers in the FI community, and in this paper we also present additional insights gained during this

\footnotetext{
${ }^{1}$ This is available online at http://fisa.future-internet.eu/index.php/ FIA_Research_Roadmap

2 See www.effectsplus.eu 
process of validation. Overall, we observe that there are several important areas of innovation for Future Internet research and that these need to be developed and supported by researchers and policymakers both within and outside Europe.

Research Priorities. In presenting the research needs in this roadmap we have brought out the 'horizontal challenges' — those that underpin most, if not all, of the applications and use of Future Internet. In developing this roadmap we have had specific inputs in the areas of smart cities, the future of enterprise, digital media, and inclusion. We had little input in areas such as healthcare, energy management, transportation. We don't think that is too much a limitation our aim has been to bring out the broad and horizontal research themes that will persist, even grow in scope and opportunity and applicability as we look past the middle of the decade, and particularly those area that address fundamental capabilities, and needs of a networked information society.

In our research priorities for the Future Internet we see three themes that are foundational and enabling and which support us in building the Future Internet. These are:

1. Going Beyond Converged Infrastructure: the Internet infrastructure beyond 2020 brings new capabilities and capacities;

2. The Rise of Networked Data: learning to exploit the Internet's natural resource;

3. Achieving Real Internet Security: maintaining the security of the Internet and its users online.

Secondly, looking forward towards the research that will transform what we do and how we do it and which are fundamentally integrative, they exploit and use a wide range of networked technologies towards a diverse set of objectives, we see three priorities that support us in using the Future Internet. These are:

4. Networked Interaction: people interacting with each other, with information, and with cyber-physical worlds;

5. Augmented Worlds: from an Internet of things to an Internet doing things;

6. Internet-style Innovation: the Internet as an innovation ecosystem, supported by architecture, policy, and invention.

Going Beyond Converged Infrastructure. Internet infrastructures - networks, virtualised computing, storage systems, undergoing a period of intense convergence, the boundaries between service platforms and the infrastructure services layer are becoming blurred, and at the same time at the edge of the networks smart mobile devices are becoming pervasive and more capable at the edges of networks.

Future research must look beyond converged infrastructure to the addressing the challenges of meeting the vastly increased demand for bandwidth and services and connectivity and new and different applications and services: 
- Polymorphic networks - combining different networks to meet the for capacity and needs of new media, applications, services, infrastructures and networked 'things'

- Expanding the cloud to the edges of the network and beyond, providing the execution environments for new FI applications and services. Real time capabilities will be vital for these new services, data and event processing, interaction processing all demand real time responses, and variability in demand will provide real challenges for the services providers

- Looking beyond smart devices towards smart edge-systems as the execution environments at the edges of the network that link the physical and the cyber world.

The Rise of Networked Data. Networked data on a massive scale is the powerhouse of the Internet today and the growth trend looks set to continue as new services and applications are developed, a greater part of the economy and public sector relies on the Internet, and citizens spend increasing parts of their lives online.

Networked data is a horizontal capability of the Future Internet. The organisation, exploitation, and governance of the huge amount of data and information in the Internet will create ongoing opportunities and challenges for the next decade. There are underlying tensions between rights of citizens, businesses, and the state over data; the opportunities opened up by integrating data from multiple sources; the need develop new data models to make sense of the myriad of applications and sources (e.g. the 3D world for augmenting spaces, of tacit knowledge in knowledge supply chains), of records of usage, of surveillance data gathered, the list continues to grow.

Achieving Real Internet Security. A decade away we will be conducting much more of our lives and economy online than we do now. The scale of Internet use continues to increase relentlessly, and our reliance on the Internet continues to increase. As we do so the potential opportunity, rewards, and impact of cybercrime becomes even more significant. The scale of threats, potential for conflict between individuals, organisations, and states online should not be underestimated. Reliance on Internet for operation of our critical infrastructures means that cyber defence is an even more vital aspect of state security; particular concerns here are cyber attacks by unfriendly states, as well as cyber interventions by activists. Cyber attacks will become industrialised, and we need to guard against organised cybercrime as well as targeted attacks on individuals or particular systems.

Securing the Internet as a socio-technical system is a high priority and continued separate attention is necessary. Internet security can become part of Europe's new defence businesses, and a secure Internet is necessary condition for economic competitiveness. It is imperative to make the Internet safe and secure, so it is a positive experience for all users, independently of their background or education. At the same time, we need to make security controls transparent and 
unobtrusive, so that they do not hinder openness and availability of network resources.

Networked Interaction. Through the Internet we interact with each other, with the physical world, and with the digital world, and indeed, in the future the distinctions my blur even further. Social networks are not the last word on social interaction, webcams and video conferencing are not the last word on collaboration, games and IPTV are not the last word on entertainment. New interfaces and modalities will create opportunities for richer interaction and for addressing our work, life and emotional needs. New ways of interacting with complex data provide ways to understand complex situation. New interactions with the digital world will provide new media experiences that look beyond $3 \mathrm{~d}$. Future networked interaction will not be delivered through one device, in a sitback, sit-up, or handheld interaction mode, but through collections of devices brought together as smart edge systems, and ideas of ownership, situatedness, virtualisation will create interaction experiences that are effective, engaging, and empowering.

The research theme of interaction, supported by rich interfaces, displays, haptics, and other yet to be developed approaches makes possible to address some of our real concrete needs too - for carbon reduction for example through remote collaboration which can take people off roads, support knowledge business networks, or create valuable social links. Some of the biggest barriers to delivering Internet benefits to excluded groups in Internet are the interfaces. This is just one example, and looking forward the future of networked interaction has real potential to create value given that we are reaching the point where demand and capability come together to make new and valuable networked interactions possible.

Augmented Worlds. The vision of networked services, systems, and devices supporting us in our work and social lives, or in business to control and manage processes and operations has been with us for some time. Hitherto, the Framework Programme Seven has explored Internet of services, reflecting the shift of our economy to a service economy, Internet of things, reflecting the opportunity to measure and manage the physical word using networked systems and these capabilities are beginning to be available.

As we look to the start of the next decade and beyond we can begin explore how we can harness the power of the Internet to augment lives, work, business and spaces in ways that add value. By 'augmentation' we mean 'increasing in intensity' the activities we are doing or the things we need done for us, addressing what we do in our jobs and daily lives, addressing needs of groups and communities, of industry, construction, maintenance, engineering, manufacturing, transport with information, decisions support, risk analysis, options, delivered through interactions and interfaces that are intuitive and unintrusive. What is currently described as augmented reality has potential to develop into what is fundamentally an integrative, systems, applied approach to addressing problems of industry, people, society and developing techniques and frameworks that 
harness the scale of the network and networked data onto individual actions, tasks, and activities, transforming what we do and how we do it.

Internet-style Innovation. It is clear that the Internet has been an incredible force for innovation over the past three decades at least. To have created such a platform for innovation, value creation, and benefits to society must rank as one of the outstanding achievements since the industrial revolution. It is our aim that this innovation and value creation should continue, and that Europe should play a big part in it. Every one of the research communities consulted in this programme stressed innovation - both within their field of work and enabling innovative benefits as a consequence of it.

Whether we are discussing topics such future enterprise, cities, or experiences, ideas abound on the kinds of approaches that enable innovative value creation to take off. The network effect, scale, openness, experimentation, software, and pilots, and services, SME and start up participation, application, and real users. This is the 'Internet-style' innovation we aim for. Innovation that happens at the edges of the network, that is stimulated by linkages between sectors, that involves people, where people, communities, business, even public sector, are 'empowered' to take control of opportunities to innovate. Ideas such as making cities into experimental services environments, creating platforms, integrating across industries and sectors, releasing and exploiting data, are enabling factors - they set the conditions for unlocking value and if carried out 'Internet-style' they set the conditions for innovation and provide an environment for new applications and services need to be instantiated, built, used, and grow.

Conclusions. We have highlighted the themes above because they need to be directly addressed and they deal with different aspects of the Future Internet. There are also a number of approaches that are essential to the success of Future Internet initiatives, where we discuss approached to Future Internet research.

As FI develops support for a wide range of stakeholders seeking to develop, provision, or use a range of networked components and concepts there needs to be an architectural framework that provides ongoing guidance, specification and rules of how systems should behave, how everything fits together, how networked elements communicate, and how elements are (dynamically) structured into larger interoperating entities. The architecture needs to present the whole picture, to relate the relevant elements in the picture, and to maintain its own forward plan or roadmap as a consistent part of the overall FIA roadmap. Such architectural coordination has typically been provided by groups such as IETF. As we look forward to research in Future Internet we emphasise the need for the research to take an architectural approach at all levels from infrastructure to services and applications, and to participate in, and where appropriate develop new forums for providing and developing that architectural coordination.

Open Access. This article is distributed under the terms of the Creative Commons Attribution Noncommercial License which permits any noncommercial use, distribution, and reproduction in any medium, provided the original author(s) and source are credited. 